\title{
对硝基苯乙烯基葸的光谱行为的研究 *
}

\author{
王 华** 张宝文 曹 怡*** \\ (中国科学院感光化学研究所光化学开放实验室, 北京 100101)
}

\section{关键词 对硝基苯乙㛓基蓦衍生物 分子内电子转移 能级近似效应}

近年来, 具有共轭 $\pi$ 电子体系的电子给体-受体 (D-A) 化合物引起了人们的极大兴 趣 ${ }^{[1-4]}$, 这些化合物能发生光诱导分子内电荷转移, 使其激发态分子的偶极矩远大于基态, 它 们的发射光谱对介质的极性、粘度、温度十分敏感, 随分子的结构变化而变化, 展现出特有的光 电性质, 可利用作为非线性光学材料 ${ }^{[3]}$, 光电转换材料以及极性、粘度的苂光探针 ${ }^{i 4]}$ 等. 因此 从分子水平上认识光诱导电子转移与电荷分离过程, 对探讨其结构与发光性质、非线性光学活 性及光能转换等性能间的关系是十分重要的.

本文从葱的不同活性位置入手, 设计合成了两个共轭的 D-A 化合物 : 2-pNSA 和 9-pNSA, 研究了介质极性与温度对化合物发光能力的影响, 考察了葱 2-位与 9-位取代化合物分子构象 上的差异与化合物发光能力的关系, 得到了一些有意义的结果, 对设计合成新的 D-A 化合物 有一定的参考价值.

\section{1 实验部分}

\section{1 合成}

（1）反式 2-对硝基苯乙烯基葱(2-pNSA)的合成：1）对硝基苄溴: $100 \mathrm{~mL}$ 圆底烧瓶中加 入 $1.3 \mathrm{~g}(9.4 \mathrm{mmol})$ 对硝基甲苯, $1.8 \mathrm{~g}(10.1 \mathrm{mmol}) \mathrm{N}$-溴化丁二酰亚胺及 $50 \mathrm{~mL}$ 四氯化碳, 回 流搅拌下加入少量过氧化苯甲酰作引发剂, 回流 $3 \mathrm{~h}$, 冷却, 过滤, 蒸出四氯化碳得黄色粗品, 对 粗品用乙醇重结晶得浅黄色针状晶: $1.7 \mathrm{~g}(82.9 \%)$. mp. 94 97C；2) 反式 2-对硝基苯乙 烯基葱 (2-pNSA)： $10 \mathrm{~mL}$ 圆底烧瓶中加入 $0.1 \mathrm{~g}(0.46 \mathrm{mmol})$ 对硝基芐澳, $0.13 \mathrm{~g}$ 三苯基膦及 $5 \mathrm{~mL}$ 干苯, 回流搅拌下, 逐渐有浅黄色沉淀产生, $3 \mathrm{~h}$ 后停止反应, 冷却, 过滤即得膦盐. 将所 得干燥的膦盐中加入 $0.1 \mathrm{~g}$ 2-葱甲醛及 $5 \mathrm{~mL}$ 无水二氯甲烷, 回流下小心加入少量乙醇钠, 反 应液呈深红色, 继续回流 $4 \mathrm{~h}$, 冷却, 用 $20 \mathrm{~mL}$ 二氯甲烷萃取, 水洗, 尔后进行两次硅胶柱层析, 石油醚 (60 90 $0^{\circ}$ )/氯仿 (3:1) 作淋洗液, 得亮黄色粉末 $50 \mathrm{mg}(33.2 \%)$.

mp. $269 \sim 272{ }^{\circ} \mathrm{C}$

${ }^{1} \mathrm{H}-\mathrm{NMR}\left(\mathrm{CDCl}_{3}\right): \delta$ 值为 $6.70 \sim 6.80(\mathrm{~d}, 1 \mathrm{H}) ; 7.00 \sim 7.10(\mathrm{~d}, 1 \mathrm{H}) ; 7.20 \sim 8.50$

1995-08-30 收稿, 1995-12-28 收修改稿

* 国家自然科学基金和国家科学技术委员会基础性研究重大课题资助项目

**95 届博士毕业生,现在河南大学化学化工系工作(开封 475001)

***联系人 
$(\mathrm{m}, 9 \mathrm{H})$;

$\mathrm{IR}\left(\mathrm{KBr}, \mathrm{cm}^{-1}\right)$ : $1605(\mathrm{C}=\mathrm{C}) ; 1589,1508$ (苯环骨架振动)；1 $335\left(-\mathrm{NO}_{2}\right)$; 965 ( $\mathrm{C}=\mathrm{C}$, 反式);

MS, $m / e: 325\left(\mathrm{M}^{+}, 100\right)$.

（2）反式 9-对硝基苯乙烯基葱 (9-pNSA)的合成: 合成方法同反式 2-对硝基苯乙烯基葱 的合成, 产率为 $53.0 \%$.

mp. $219 \sim 2200^{\circ}$

${ }^{1} \mathrm{H}-\mathrm{NMR}\left(\mathrm{CDCl}_{3}\right): \delta(7.30 \sim 8.60)$ (共轭 $\pi$ 电子体系的 $\mathrm{H}$ ).

\section{2 测试仪器}

${ }^{1} \mathrm{H}-\mathrm{NMR}$ 谱在 Varian $300 \mathrm{MHz}$ 核磁共振谱仪上测试, 红外谱在 Perkin-Elmer 983G 型红 外光谱仪上测试, 质谱在 Finnigan GC-MS 4021C 型质谱仪上测试. 实验中所有的吸收光谱、 苂光光谱分别在 Hitachi 330 型紫外-可见分光光度计及 Hitach MPF-4 型荧光光谱仪上测得, 光谱测试溶剂为分析纯, 测量前对样品通 $\mathrm{N}_{2} 20 \mathrm{~min}$.

\section{2 结果与讨论}

\section{1 吸收光谱行为}

图 1 给出了 2-pNSA 与 9-pNSA 于氯仿中的吸收光谱, 从图中可以看出 9-pNSA 的长波方 向上的吸收峰无精细结构, 而 2-pNSA 的长波方向上的吸收峰似乎有精细结构, 在 $410 \mathrm{~nm}$ 处左 右有一弱的肩峰; 其峰位较 9-pNSA 也大约有 $60 \mathrm{~nm}$ 的紫移, 相比之下 9-pNSA 的长波吸收带 较为宽散, 表明分子内较 2-pNSA 存在有显著的电荷转移 (CT) 相互作用.

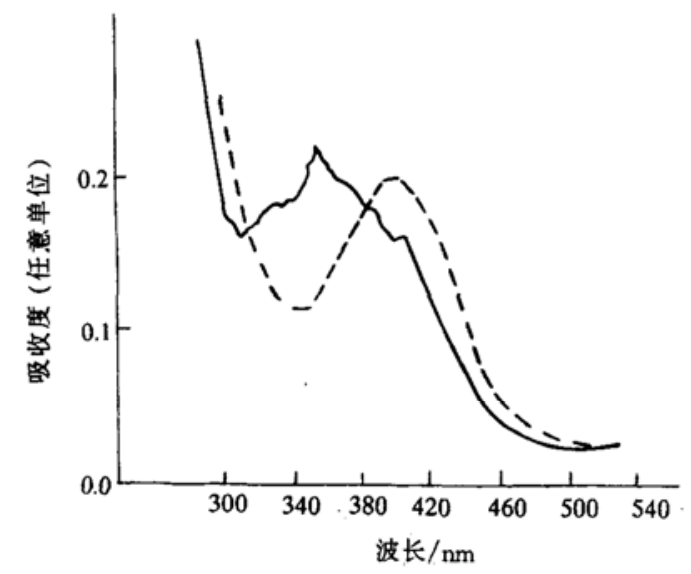

图 12 2-pNSA(- - , 9-pNSA(-.-) 在氯仿中的吸收 光谱 $[\mathrm{C}]=1 \times 10^{-5} \mathrm{~mol} / \mathrm{L}$

Becker ${ }^{[5]}$ 用 X-行射法测得 9-苯乙烯基葱 (9-styrylanthracene) 分子中苯环与葱环处于顺 式与反式时的二面角分别为 $79^{\circ}$ 与 $66^{\circ}$. Tachon 等 ${ }^{[6]}$ 研究了反式-1, 2-二(2'菜基) 乙烯 时指出其两种旋转体 (rotamer) 中葲环与乙烯 基的二面角为 $8^{\circ}$ 与 $20^{\circ}$. 由以上事实可见, 萝 2-位与 9-位衍生物分子的平面性存在很大差 异:2-位衍生物分子平面性非常明显地好于 9位衍生物分子的平面性. 2-pNSA 的吸收峰存 在精细结构可能与其有两种旋转体有关 ${ }^{[7]}$. 光谱测试表明, 在不同极性溶剂中, 该二化合 物的吸收光谱行为变化不大 (表 1,2 所示).

此外, 9-pNSA 只有在非极性溶剂 (甲苯) 中存在反式 $\rightarrow$ 顺式的光诱导分子内顺-反异构 化反应 ${ }^{[8]}$. 一些文献报道 ${ }^{[9 \sim 11]}$, 从葱 2-位相 连接的共轭化合物不能发生其反式到顺式的 光诱导分子内异构化反应, 本工作对 2-pNSA (反式) 在极性与非极性溶剂中在直接光照与加 入三线态敏化剂的条件下,均没有观察到分子内顺-反异构化反应. 


\section{2 苂光光谱行为}

（1）溶剂效应：从表 1 可见 2-pNSA 随着溶剂极性的增大其荧光发射峰位大幅度红移, 从 环已烷中的 $460 \mathrm{~nm}$ 到二氯甲烷中的 $620 \mathrm{~nm}$ 红移了 $160 \mathrm{~nm}$, 而荧光发射强度 $\left(\Phi_{\mathrm{f}}\right)$ 呈现先增强 后减弱的有趣的抛物线变化 (参见表 1 及图 2). 这种现象的出现表明 2-pNSA 激发态下的发 光受两种机制共同制约: 分子内电子转移机制与能级近似效应.

表 12 -pNSA 于不同极性介质中的光谱参数 ${ }^{a)}$

\begin{tabular}{lccccc}
\hline \multicolumn{1}{c}{ 溶剂 } & $\begin{array}{c}E_{\mathrm{T}}(30) \\
/ \mathrm{J} \cdot \mathrm{mol}^{-1}\end{array}$ & $\begin{array}{c}\lambda_{\max }^{\mathrm{ab}} \\
/ \mathrm{nm}\end{array}$ & $\begin{array}{l}\lambda_{\max }^{\mathrm{f}} \\
/ \mathrm{nm}\end{array}$ & $\begin{array}{l}v_{\mathrm{a}}-v_{\mathrm{f}} \\
/ \mathrm{cm}^{-1}\end{array}$ & $\Phi_{\mathrm{f}}$ \\
\hline 环已烷 & 130.5 & 353 & 460 & 6589 & 0.015 \\
四氧化碳 & 136.0 & 355 & 490 & 7761 & 0.072 \\
苯 & 145.2 & 360 & 500 & 7778 & 0.20 \\
四氢呋喃 & 156.9 & 360 & 550 & 11276 & 0.099 \\
氧仿 & 163.6 & 360 & 606 & 9596 & 0.022 \\
二氧甲烷 & 172.0 & 360 & 620 & 11649 & 0.006 \\
\hline
\end{tabular}

a) 以硫酸奎宁的 $0.1 \mathrm{~mol} / \mathrm{L}$ 稀硫酸溶液作为苂光标准物 [12]

激发态下 D-A 化合物易发生分子内的电 子转移与电荷分离, 达到所谓的分子内电荷转 移 (ICT) 态, 产生 ICT 态苂光. 而电荷的转移 使得分子激发态的偶极矩远大于基态, 介质极 性的增加对分子的激发态起到了稳定化的作 用. 在大极性介质中, 当激发态 D-A 分子发生 了分子内电子转移与电荷分离从而产生正负 双极离子, 使得分子发光的能力减弱甚至丧 失. 故光致分子内电荷转移机制导致宏观上 随介质极性的增加, 激发态 D-A 化合物发光峰 位的红移与发光强度的下降.

另一方面, 对含有杂原子 $(O, N$ 等) 的杂环 体系化合物与芳香共轭体系化合物往往存在 能级近似效应 ${ }^{[13,14]}$, 即这类化合物能量最低 的 $n \pi^{*}$ 态与能量最低的 $\pi \pi^{*}$ 能级非常近似, 往往导致从 $n \pi{ }^{*}$ 和 $\pi \pi^{*}$ 中能量较低的一个激 发态的有效的无辐射失活. 随着介质极性的 增加, $S_{1}\left(\pi \pi^{*}\right)$ 与 $S_{2}\left(n \pi^{*}\right)$ 间的能级间隔增

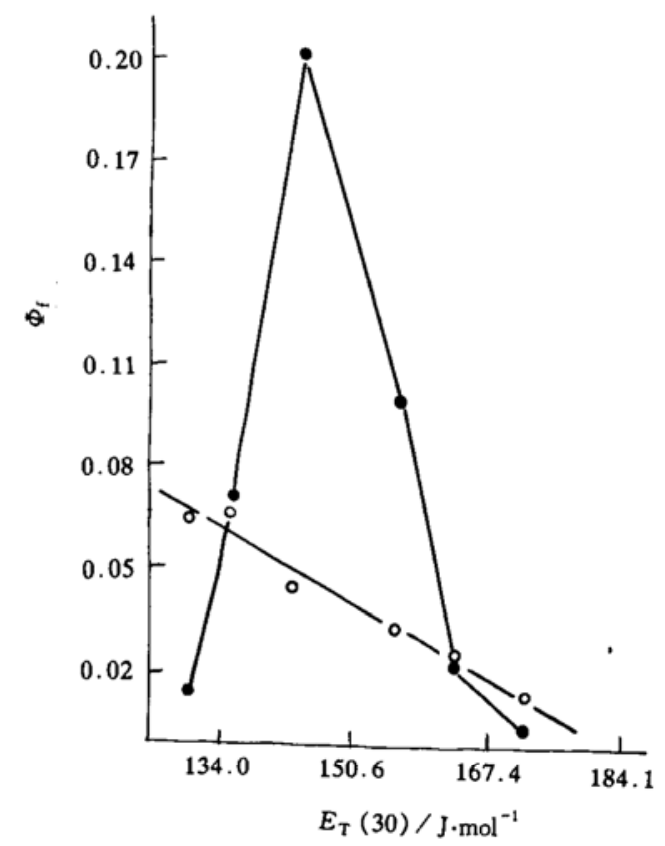

图 2 2-pNSA( ( ) 与 9-pNSA(o) 的 $\Phi_{\mathrm{f}}$ 随介质极性 参数 $E_{\mathrm{T}}(30)$ 的变化关系 加 (参见图 3), 能级近似效应减弱, 荧光发射强度 $\left(\Phi_{\mathrm{f}}\right)$ 增加, 所以能级近似效应可导致激发态 分子发光能力随介质的极性增大而增大的趋势.

正是由于分子内电子转移机制与能级近似效应对激发态 D-A 分子发光能力的相互竞争, 导致了 2-pNSA 的发光强度随介质极性增大而出现先增强后减弱的抛物线式的变化趋势. 


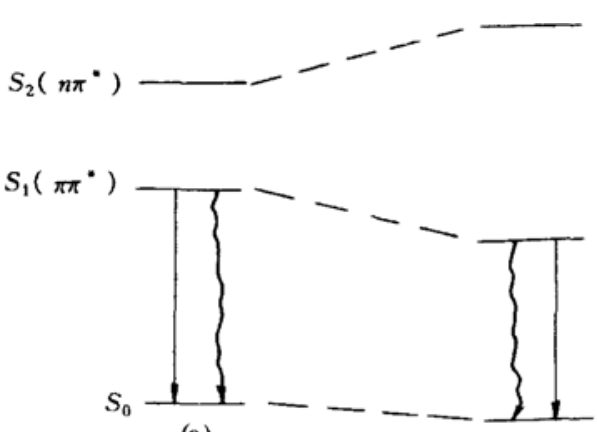

(a)

图 3 能级近似效应示意图 （a）非极性溶剂中，（b）极性溶剂中

与 2-pNSA 不同, 9-pNSA 在不同极性介质 中均为单峰发射, 其发光强度随介质极性的增 加而下降 (如图 2, 表 2 所示), 说明了控制激 发态下 9-pNSA 发光的机制主要是分子内电荷 转移机制, 而能级近似效应的作用显得较弱. 2-pNSA 与 9-pNSA 分子内硝基苯与葱环间的共 轭程度不同, 9-pNSA 分子的平面性较差, 分子内 电荷转移过程较弱, 但其电荷复合 (charge recombination) 过程也差, 电荷转移后的电荷分离 态较为稳定, 其 ICT 态能级较低. 2-pNSA 分子 的平面性较好, 分子内电荷转移与电荷复合过 程均容易发生, 反而不利于电荷分离态的形成,

其 ICT 态能级比 9-pNSA 的要高. 故在不同极性溶剂中, 2-pNSA 的苂光最大发射峰位均在 9-pNSA 的相应发射峰位的短波方向.

表 2 9-pNSA 于不同极性介质中的光谱参数 ${ }^{2}$

\begin{tabular}{lccccc}
\hline \multicolumn{1}{c}{ 溶剂 } & $\begin{array}{c}E_{\mathrm{T}}(30) \\
/ \mathrm{J} \cdot \mathrm{mol}^{-1}\end{array}$ & $\begin{array}{c}\lambda_{\max }^{\mathrm{ab}} \\
/ \mathrm{nm}\end{array}$ & $\begin{array}{l}\lambda_{\max }^{\mathrm{f}} \\
/ \mathrm{nm}\end{array}$ & $\begin{array}{l}v_{\mathrm{a}}-v_{\mathrm{f}} \\
/ \mathrm{cm}^{-1}\end{array}$ & $\Phi_{\mathrm{f}}$ \\
\hline 环已烷 & 130.5 & 396 & 496 & 5091 & 0.064 \\
四氯化碳 & 136.0 & 404 & 515 & 5335 & 0.065 \\
苯 & 145.2 & 403 & 538 & 6227 & 0.045 \\
四氢呋哺 & 156.9 & 401 & 575 & 7546 & 0.035 \\
氯仿 & 163.6 & 404 & 636 & 9029 & 0.0027 \\
二埭甲㜔 & 172.0 & 404 & 645 & 9249 & 0.0013 \\
\hline
\end{tabular}

a) 以硫酸奎宁的 $0.1 \mathrm{~mol} / \mathrm{L}$ 稀硫酸溶液作为荧光标准物 ${ }^{[12]}$

从表 2 可见, 9-pNSA 的苂光随介质极性增加其发射峰位也发生了大幅度的红移, 从环己 烷中的 $496 \mathrm{~nm}$ 到二氯甲烷中的 $645 \mathrm{~nm}$, 红移了约 $150 \mathrm{~nm}$. 说明了激发态下 9-pNSA 于极性 溶剂中可发生强烈的分子内电子转移与电荷分离.

(2)温度效应：2-pNSA 在不同温度下于 2-甲基四氢呋喃中的苂光光谱(图略). 随介质 温度的降低苂光发射峰位红移(详见表 3) 而发射强度变化不大, 只有到冻结态 (77 K) 下, 其荧 光发射强度才有大幅度增加.

流动相下, 随介质温度的降低 2-pNSA 的苂光发射强度变化不大, 这一现象可能是由以下 3 种因素共同作用的结果: 第一, 随介质温度的降低, 溶剂的极性有所增加 ${ }^{[15]}$, 极性诱导下分 子内电子转移与电荷分离的能力增强, 导致苂光发射峰位红移与苂光量子产率 $\left(\Phi_{i}\right)$ 下降的趋 势. 第二, 随介质温度的降低, 溶剂的极性增强, 能级近似效应减弱, 而能级近似效应的减弱使 得分子的荧光发射强度有增强的趋势. 第三, 随介质温度的降低, 各种非辐射失活过程逐渐减 弱, 易使得分子的发光过程增强, 分子的苂光量子产率有增大的趋势. 正是由于这几种因素的 共同影响导致了 2-pNSA 的发光强度随介质温度的降低而变化不大. 
表 3 2-pNSA 与 9-pNSA 在不同温度下于 2-甲基四氢呋喃中的荧光光谱数据

\begin{tabular}{lccccc}
\hline$T / \mathrm{C}$ & $\mathrm{RT}$ & -23 & -63 & -94.7 & $77 \mathrm{~K}$ \\
\hline $2-\mathrm{pNSA}$ & & & & 580 & 500 \\
$E_{\max } / \mathrm{nm}$ & 535 & 550 & 560 & 0.85 & 7.80 \\
相对 $\Phi_{\mathrm{f}}$ & 1.00 & 0.82 & 1.02 & & \\
\hline $9-\mathrm{pNSA}$ & & 585 & 600 & 605 & 514 \\
$E_{\max } / \mathrm{nm}$ & 574 & 1.23 & 1.70 & 2.02 & 6.39 \\
相对 $\Phi_{\mathrm{f}}$ & 1.00 & & & & \\
\hline
\end{tabular}

当介质的温度降低到 $77 \mathrm{~K}$ 时, 溶质分子与溶剂分子均被冻结, 介质的溶剂化作用已变得 很小, 各种非辐射失活过程均被消除, 故此时 2-pNSA 的荧光发射来自 Franck-Condon 激发态, 其峰位大范围蓝移, 荧光发射强度大幅度升高.

与 2-pNSA 不同, 9-pNSA 的苂光量子产率随介质温度降低呈上升趋势 (图略, 表 3)。上 已述及, 9-pNSA 分子发光受能级近似效应影响很小, 故随介质温度降低、介质极性的增加使 得 9-pNSA 的发光红移, 苂光量子产率 $\left(\Phi_{\mathrm{f}}\right)$ 有下降的趋势. 另一方面, 由于介质极性的增加各 种非辐射失活过程迅速减小, 菼光量子产率 $\left(\Phi_{\mathrm{f}}\right)$ 升高的趋势较为突出. 从室温到 $-94.5^{\circ} \mathrm{C}$ 的 温度变化范围内, 2-pNSA 红移了 $45 \mathrm{~nm}$ 而 9-pNSA 红移了 $31 \mathrm{~nm}$, 可见 2-pNSA 受介质极性变 化的影响大于 9-pNSA 所受的相应影响, 故温度效应影响 2-pNSA 的发光主要因素是介质的 极性, 而温度影响 9-pNSA 的发光主要因素是非辐射失活过程, 即其随介质温度的降低而迅速 减弱, 介质的极性变化对其发光能力的影响较弱. 到冻结态 $(77 \mathrm{~K})$ 下, 随着非辐射失活过程的 大大减弱, 溶剂化作用的消失, 9-pNSA 的发光大幅度蓝移, 苂光量子产率 $\left(\Phi_{\mathrm{f}}\right)$ 大幅度升高(表 3).

从表 3 还可以看出, 冻结态 $(77 \mathrm{~K})$ 下 $2-\mathrm{pNSA}$ 的苂光最大发射峰位 $\left(E_{\max }=500 \mathrm{~nm}\right)$ 小于 9-pNSA 的最大发射峰位 $\left(E_{\max }=514 \mathrm{~nm}\right)$, 说明 2-pNSA 的 CT 激发态的能量高于 9-pNSA 的 $\mathrm{CT}$ 激发态的能量, 而该二化合物的区别仅在于葱环上的连接位置不同, 正因为与葱环的连接 位置上的差异造成了分子平面性质的不同. 冻结态是被认为分子可以保持其基态下的构象, 故平面性较差的 9-pNSA 其激发态下的分子内电荷转移过程弱于 2-pNSA 的相应过程, 但其 后的电荷复合过程也较差, 可形成较为稳定的电荷分离态. 故与 2-pNSA 相比, 9-pNSA 的 CT 的荧光发射峰位在较长波长方向上.

综上所述, 2-pNSA 与 9-pNSA 的发光性质对介质的极性与温度的变化十分敏感, 呈强烈 的依赖性. 溶剂极性效应显示了 2-pNSA 的分子内电荷转移( ICT) 态的发光受分子内电子转 移机制与能级近似效应的共同制约, 其苂光量子产率 $\left(\Phi_{\mathrm{f}}\right)$ 随介质极性的增加呈抛物线式的有 趣变化. 而 9-pNSA 的发光受能级近似效应的影响很弱, 其苂光量子产率随介质极性的增加 呈线性减弱的趋势.

从该二化合物对介质温度的依赖关系来看, 分子的构象的差异是影响分子发光强度与发 射峰位的主要因素. 


\section{参考文献}

1 Rettig W. Photophysical and photochemical switches based on twisted intramolecular charge transfer (TICT) states. Appl Phys, 1988, B45:145

2 Wang $\mathrm{Y}$. Quenching of singlet and triplex state of p-N, N-dimethyl-aminobenzonitrile by tertiary amines: intromolecular charge-transfer state and excited-state three-electron bond. J Chem Soc, Faraday Trans 2, 1988, 84(11): 1809

3 Sing D K, Sohn J E. Second-order nonlinear-optical properties of donor- and acceptor-substituted aromatic compounds. J Opt Soc Am B, 1989, 6: 1339

4 Loufty R O. Fluorescence probes for polymer free-volume. Pure Appl Chem, 1986, 58: 1239

5 Becker H D, Patrick V A, White A H. Molecular geometry of (E)- and (Z)- ( $\beta$-styryl) anthracenes. Aust J Chem, 1984, 37: 2215

6 Tachon M, Davies E, Lamotte M. Shpolskii spectra of trans-1, 2-di (2'-pyridyl) ethylene identification and evidence for almost planar structure and $\pi$ type HMO for the trapped rotamer. J Phys Chem, 1984, 98: 11870

7 Arai T, Karatsu T, Sakuragi $\mathrm{H}$ et al. Highly selective rotational isomerization of 2-vinylanthracene in the excited singlet state picosecond time resolved fluorescence study. Chem Phys Lett, 1989, 58(5): 429

8 李文杰,张宝文,曹 怡. 硝基苯乙烯基芳香化合物光谱性质的研究. 中国科学, B 辑, 1994, 24(1): 1 6

9 Arai T, Karatsu T, Sakuragi H et al. One-way photoisomerization between cis- and trans-olefin: a novel adiabatic process in the excited state. Tetrahedron Letters, 1983, 2873

10 Karatsu T, Arai T, Sakragi H et al. New insight into one way photoisomerization of anthrylenes: absorption spectra of the trans triplets as intermediates of the isomerization. Chem Phys Lett, 1985, 115(1): 9

11 Arai T, Tokumaru K. Photochemical one-way adlabatic isomerization of aromatic olefins. Chem Rev, 1993, 93:23

12 Demas J N, Crosby G A. The measurement of photoluminescence quantum yields: a review. J Phys Chem, 1971, 75(8): 991

13 Lai T I, Lim B T, Lim E C. Photophysical properties of biologically important molecules related to proximity effects: psoralens. J Am Chem Soc, 1982, 104: 7631

14 Siebrand W, Zgierski M Z. Radiationless decay of vibronically coupled electronic states III. Strong coupling and its effect on triplet decay in aromatic hydrocarbons. J Chem Phys, 1981, 75: 1230

15 van Damme M, Hofkens J, De Schyver F C. Solvent dynamics and intramolecular charge transfer in 4-cyano-4'-butyloxybiphenyl (4COB). Tetrahedron, 1989, 45(15): 4693 